\title{
Childhood Domestic Physical Violence Abuse and Emotional Violence Abuse Negative Effects on Adult Mental Health
}

\author{
JinYi Chen ${ }^{1, *}$ \\ ${ }^{I}$ Education Department, University of York, Heslington, United Kingdom \\ *Corresponding author. Email: jc3363@york.ac.uk
}

\begin{abstract}
Childhood adversity or adverse childhood experience (ACE) is a common experience for many children. As one of the adverse childhood experiences, domestic violence has serious effects on child development and adult health. This review divides domestic violence into two components: physical and emotional domestic violence abuse, and analyzes the effects of each type of domestic violence on mental health in adulthood. This study also compares the interventions and treatments proposed by current research and suggests directions that future research could explore based on past literature related to domestic violence and mental health.
\end{abstract}

Keywords: domestic violence, adulthood, intervention, mental health

\section{INTRODUCTION}

More than half of all children suffer at least one adverse or traumatic experience ${ }^{[2]}$. Previous studies have shown that childhood adversity or adverse childhood experiences often include child abuse such as physical abuse, emotional abuse, sexual abuse, physical neglect, emotional neglect or changes in family structure (divorce, death of a loved one, substance abuse by a family member, etc.) ${ }^{[1]}$. A large body of researches on childhood adverse experiences (ACEs) have confirmed that emotionally disturbed adults have always experienced adverse childhood experiences, including sexual or physical abuse, emotional abuse and neglect, and witnessing domestic violence. Under chronic ACEs, children's brains and bodies produce excessive stress hormones, which impair their brain function. In the long term, those can alter their response patterns in life, leading to chronic illnesses such as heart disease or depression in adulthood, as well as an increased likelihood of being a victim of violence. The researches on ACEs currently has consensus in at least three perspectives. First, it recognizes that different antecedent risks may lead to similar outcomes and that these risks are often interrelated. Thus, individuals who experience one kind of ACEs are often exposed to multiple ACEs. Second, ACEs often have a dose-response relationship with many undesired outcomes; that is, the accumulation of adversity usually leads to graded decrements in development and function across domains. Third, ACEs can lead to lifelong consequences. It is now widely accepted that early adversity leads to morbidity and mortality throughout the life course, although the mechanisms affecting long-term outcomes are unclear ${ }^{[1][6][9][12]}$. In summary, these experiences have detrimental effects on both physical and mental health of children.

Domestic violence accounts for the majority of childhood adversity or ACEs ${ }^{[1]}$. National and international research studies have found that domestic violence experienced in childhood or witnessing violence between parents leads to increased risk of depression $^{[2][5][8]}$, suicide ${ }^{[3][5]}$, alcoholism, and drug addiction in adulthood ${ }^{[3][4][6]}$. Domestic violence is generally divided into two typesof abuse: physical abuse (childhood physical abuse) and emotional abuse (childhood emotional abuse) . Childhood physical abuse (CPA), also known as 'hard violence' ${ }^{[26]}$, which refers to children's witnessing of violent behavior between parents and parental violence against children. CEA can be classified as emotional neglect (indifference to the child's emotional needs or indifferent treatment between parents), emotional denial (excessive sarcasm, sarcasm, reprimands, and other forms of verbal abuse toward the child or between parents), and over-involvement (forcing beliefs on children). 
Almost all existing literature discusses the impact of different types of domestic violence or ACEs on mental health and functioning ${ }^{[1][2][4-10][23]}$ or interventions that address the impact of childhood trauma ${ }^{[16-18]}$, as well as larger dimensions ${ }^{[21]}$. There is no comprehensive review that integrates the effectiveness of domestic violence interventions, and the existing literature does not separately discuss the effects of childhood emotional violence (CEA) and childhood physical violence (CPA) on mental health in adulthood. This literature review will analyze the effects of two different forms of domestic violence on adult mental health and analyze the current interventions. Future research can explore more effective interventions to address the psychological trauma of childhood domestic violence.

This review is divided into two parts: the effects of childhood physical family abuse on mental health in adulthood, and the effects of childhood emotional family abuse on mental health in adulthood. The purpose of this review is to explore the mental health in adulthood with experiences of childhood physical or emotional abuse, respectively, and to make recommendations for future studies. Negative mental health and personality development effects resulting from parental separation or other ACEs will not be discussed in this review.

\section{EFFECTS OF CHILDHOOD PHYSICAL DOMESTIC VIOLENCE ABUSE ON MENTAL HEALTH IN ADULTHOOD}

Childhood physical domestic violence (CPA) includes violencesuch as slapping with hands or objects, spanking, kicking, throwing, and burning children ${ }^{[2][3]}$. Individuals who experience behavioral violence in childhood are at significantly increased risk for psychological disorders in adulthood, including personality disorders, schizophrenia ${ }^{[2]}$, depression, anxiety, and fears ${ }^{[1][2][3]}$, etc. Rich et al. evaluated 551 college women's childhood abuse history, traumatic experiences and depression, and interpersonal skills ${ }^{[25]}$. The results showed that women were more likely to be victims of sexual assault or domestic violence in adulthood if they had experiences of childhood or adolescent physical or sexual abuse, and that physical and emotional violence such as verbal abuse were positively associated with depressive tendencies. In addition, they also found that father's verbal abuse predicted psychological trauma and mother's verbal abuse predicted relationship problems. $\mathrm{Lu}$ et al. examined the relationship between severe mood disorders and ACEs ${ }^{[4]}$. The results showed that adults with severe mood disorders were more likely to have experienced a range of ACEs. Among the most common experiences were witnessing domestic violence and physical abuse, followed by sexual abuse, being in foster care or kinship care, etc.
In addition, longitudinal studies have shown that CPA has adverse psychological effects in adulthood. A study by Springer et al. on middle-aged men and women showed that $11.4 \%$ of respondents had experienced physical abuse by their parents, and that childhood physical abuse had a graded increasing trend for depression, anxiety, anger, physical symptoms, and medical diagnoses ${ }^{[6]}$. They also found that these impacts will be subdued with aging, gender and family background, but cannot be eliminated. Fergusson, Boden, and Horwood conducted a longitudinal study of a non-selective birth cohort of 1265 children born in 1977 in the Christchurch birth area and explored the link between their mental health problems in adulthood and the sexual and physical abuse they experienced as children ${ }^{[5]}$. The results showed that young people exposed to sexual and physical abuse in childhood were at significantly increased risk of developing psychiatric disorders, including depression, anxiety, antisocial personality disorder, and suicidal ideation, after the age of 16-25. Young people who experienced childhood physical abuse were 1.5 times more likely to have a psychological disorder than those who did not experience childhood physical abuse.

The adverse effects of CPA on adulthood were also validated on a Chinese subject population. Using meta-analysis, Patrick et al. analyzed 22 research studies to examine the relationship between physical abuse and mental health in a Chinese population ${ }^{[24]}$. In the meta-analysis of 22 cross-sectional studies, a significant relationship between physical violence and poor psychological outcomes was found, manifested by a wide range of Axis I disorders such as PTSD, anxiety and depression, and Axis II personality disorders. Overall the adverse effects of abusive experiences of Chinese subjects were roughly comparable to those in the West.

\section{EFFECTS OF CHILDHOOD EMOTIONAL DOMESTIC VIOLENCE ABUSE ON MENTAL HEALTH IN ADULTHOOD}

CEA can be as traumatic as physical abuse, if not more so, because it can impede the need for love, self-esteem, and belonging ${ }^{[2][5]}$.Emotional violence and abuse (CEA) includes taunting, verbal abuse, devaluation, and neglect of the child's emotional needs ${ }^{[2][3]}$. CEA in the family often occurs in conjunction with other forms of abuse or violence, and the impact of emotional violence and abuse on children's mental health can rise to the level of cognitive disorders and suicidal ideation, as well as the potential for co-occurring social phobias [1][3][9][25]. Early experiences of emotional violence and abuse will affect the mental health of children for generations ${ }^{[7]}$. 
To understand the relationship between emotional abuse and depression, Chapman et al. set up an experiment and used the cumulative stressor model (CSP) ${ }^{[3]}$. The results showed a significant relationship between emotional abuse and a lifetime history of depressive disorder or recent depressive disorder in the female population. For males, childhood emotional abuse was the greatest risk factor for lifetime and recent depressive disorders. Studies have also shown that the accumulation of ACEs, particularly emotional abuse or neglect, significantly increased the risk of developing depressive disorders. Most importantly, emotional abuse can be combined with other forms of abuse. This shows its strongest association with depressive symptoms. In addition participants who experienced one ACE also experienced two or three other ACEs, a pattern of high co-occurrence consistent with findings in the general population and with the experimental findings of Rich et al. ${ }^{[25]}$ and $\mathrm{Lu}$ et al. ${ }^{[4]}$. Rich et al. also found that early emotional abuse predicted that children were more likely to experience intergender violence in adulthood and that family emotional abuse may be more destructive than physical abuse ${ }^{[25]}$. Gibb, Chelminski and Zimmerman explored the relationship between childhood maltreatment and depression ${ }^{[8]}$. The results showed that compared with child physical or sexual abuse, child emotional or affective abuse was more strongly related to depressive disorders. In addition, they observed the same relationship in the diagnosis for social phobia. This suggests that relative to physical or sexual abuse, family emotional violence and abuse are specific for the diagnosis of major depressive disorders and social phobia. In addition, other studies have shown that domestic emotional violence and abuse may also cause children to be cognitively susceptible to depression, and this is because in emotional abuse, the abuser directly predisposes the child to be cognitively depressed ${ }^{[8]}$.

In addition to depression and social fears, domestic CEA has implications for other psychological problems in adulthood, such as substance abuse. A study by Mersky, Topitzes, and Reynolds demonstrated that the likelihood of poor health, poor mental health, and substance abuse increases in early adulthood as the number of cumulative experiences of emotional violence increases [11]. Other literature related to domestic emotional violence and abuse suggests that CEA leads to low self-esteem, helplessness, and shame that children exhibit in adulthood, and that these effects are more far-reaching than the consequences of physical violence and abuse ${ }^{[12][13][14]}$

\section{CURRENT INTERVENTIONS AND TREATMENTS}

This review of the current literature on childhood domestic violence suggests that violence, in any form, can have a negative impact on children and may affect them throughout their lives. Factors at the individual, family, community, and broader macro-systemic levels all influence the resilience of children following abusive experiences ${ }^{[16]}$. Effective interventions based on these factors will have a progressive healing effect on the trauma of abused children.

Fortunately, even though ACEs are associated with many adverse mental health symptoms, adults can mitigate the negative effects of ACEs, an ability known as resilience. Adults must first be aware and conscious of the negative effects of ACEs and have a strong belief that the self can heal childhood trauma through meditation, emotional- focused therapy, and other types of professional assistance. These two approaches have been extensively tested and proven to be effective for psycho-emotional trauma ${ }^{[16][17]}$.

Given the pathological care received by poorly treated children, abused children may have negative expectations about the availability and trustworthiness of relationships and develop feelings of worthlessness [16]. Given the adverse sequelae of child maltreatment, relational interventions become an important way to improve adaptive developmental outcomes, especially for infants and young children [17]. Toth and Manly proposed that Child-Parent psychotherapy (CPP), an intervention that provides a supportive environment that helped to improve children's first 5 years of life The CPP model helps parents protect and care for their children's abilities by processing emotions, attending to children's emotional needs, and supporting the development of positive relationships and attachment security in ways that do not merely teach the child's skills in a didactic manner ${ }^{[18]}$. There is evidence, however, that many parents' attitudes toward such forward-looking interventions are often passive and reactive ${ }^{18]}$. In addition to CPP, Stevenson compiled 68 articles to explore the outcomes of psychological treatment on trauma of abused children, including interventions for parents, interventions for sexually abused children, and healing interventions for the mental health effects of ACE [21]. Unfortunately, the results suggest that there is no single authoritative and practically effective intervention that has the desired outcome for children who have experienced ACEs. Moreover, the number of people providing treatments for child abuse is relatively small, and only a small percentage of families have access to appropriate treatments ${ }^{[19]}$.

On a larger dimension, Moxley, Squires and Lindstrom $^{[22]}$ proposed four systems coordinating approaches targeting families to society: (1) an integrated approach through systems coordination (2) gaining greater knowledge of parent training principles for early interventionists (3) increasing the level of relationship-based services specifically for child 
welfare-related families, and (4) early interventionists and additional professional development strategies for child protection caseworkers. However, it is clear that this measure requires additional professional training and specialized research to understand the unique needs of children and families.

\section{CONCLUSION}

All of the studies that discussed in this review confirm the same points. First, whether it is child physical violence or child emotional violence, these will have a negative impact on children's mental health in adulthood and even decades later. Second, the number of ACEs a child experiences is positively associated with the likelihood of depression, anxiety, or other mental health disorders in adulthood. And participants who experienced one ACE also experienced two or three other ACEs. Finally, whether physical or other forms of violence against children always occurs in conjunction with emotional violence, meaning that children who have experienced ACEs must have experienced emotional violence.

In this area of adverse childhood experiences, researchers often use individual retrospective questionnaires or interviews as research methods, so the overall effect can be somewhat biased. With the exception of two longitudinal studies on the effects of childhood physical violence on children's mental health ${ }^{[5][6], ~ t h e ~ r e s t ~ o f ~ t h e ~ l i t e r a t u r e ~ i s ~ c r o s s-s e c t i o n a l ~}$ retrospective. Therefore it is difficult to determine the exact separate measured relationships between different physical health manifestations such as depression, anxiety and different ACEs.

In addition, there are a number of reasons why researchers must consider children's experiences of abuse more broadly. First, because there is insufficient evidence of the effectiveness of current intervention treatment modalities for abused children, it is not possible to definitively indicate whether treatment is effective or which treatment works best. Second, once immediate treatment with an abusive family has taken place, it is difficult to say whether the medium-term to long-term psychological problems of abused children differ from those of other children with similar symptoms but different etiologies. In this regard, it is clear that it may lead to consideration of the medium-term to long-term psychological difficulties of the abused child or adolescent, and that special forms of treatment are required. We may preferably need to consider it as a complex problem without regard to its specific etiology ${ }^{[19]}$.

In addition, childhood maltreatment affects children's psychological, social, and physical functioning, so early intervention is to make positive developmental domain more likely critical ${ }^{[16]}$.
Maltreatment is a heterogeneous phenomenon, and research investigations require rigorous and precise definitions of maltreatment, while more targeted prevention and intervention strategies for different manifestations and developmental sequelae can be developed through dimensions such as subtypes, developmental timing, severity, separation, and perpetrators. To fully understand the impact of maltreatment experiences on different developmental stages, these investigations should include the entire lifespan from infancy to adulthood. Studying these sequelae across neurobiological and psychological domains through multilevel investigations will provide new opportunities to understand their interrelationships, and these investigations should examine resilience as well as psychopathology to inform ways to potentially build resilience [16]. Treatment approaches should consider mediation and conciliation interventions to enhance effective interventions for key components of our position, and what works best for them. Although CPP has been evaluated in diverse populations, evaluation studies of treatment programs should continue to expand the populations in which interventions are implemented, including population characteristics that can identify more precise and sensitive intervention strategies that will produce more successful outcomes. There is an urgent need for more fully designed studies of treatment outcomes for abused children, particularly addressing the prevention of long-term sequelae of child maltreatment.

For future studies, first researchers can identify personality traits that distinguish adults who have experienced child abuse and do not exhibit depressive tendencies from those who do, as a way to more accurately examine the relationship between the potential depressiveness of abused children and adulthood. In addition, there is evidence that emotional violence and abuse are more closely related to the presence of occupational depression and social phobia in adulthood than physical or sexual abuse. Further researches are needed to test whether emotional abuse actually contributes to the development of these disorders. Given the evidence that social phobia itself is a contributor to the development of depression [15] longitudinal studies should focus on the potential sequential development of these two disorders in individuals with a history of childhood emotions. Finally, future researches need to further accurately explore the impact of early childhood experiences on subsequent mental health functioning by analyzing longitudinal data as well as controlling for types of violence.

\section{REFERENCES}

[1] Merrick, M. T., Ports, K. A., Ford, D. C., Afifi, T. O., Gershoff, E. T., \& Grogan-Kaylor, A. (2017). 
Unpacking the impact of adverse childhood experiences on adult mental health. Child Abuse \& Neglect, 69, 10-19. retrieved from https: //doi.org/10.1016/j.chiabu.2017.03.016

[2] Perez, N. M., Jennings, W. G., Piquero, A. R., \& Baglivio, M. T. (2016). Adverse Childhood Experiences and Suicide Attempts: The Mediating Influence of Personality Development and Problem Behaviors. journal of Youth and Adolescence, 45(8), 1527-1545. Retrieved from https://doi.org/10.1007/s10964-016-0519-x

[3] Chapman, D. P., Whitfield, C. L., Felitti, V. J., Dube, S. R., Edwards, V. J., \& Anda, R. F. (2004). Adverse childhood experiences and the risk of depressive disorders in adulthood. Journal of Affective Disorders, 82(2), 217-225. Retrieved from https://doi.org/10.1016/j.jad.2003.12.013

[4] Lu, W., Mueser, K. T., Rosenberg, S. D., \& Jankowski, M. K. (2008). Correlates of adverse childhood experiences among adults with severe mood disorders. Psychiatric Services , 59(9), 1018-1026. Retrieved from https://doi.org/10.1176/ps.2008.59.9.1018

[5] Fergusson, D. M., Boden, J. M., \& Horwood, L. J. (2008). Exposure to childhood sexual and physical abuse and adjustment in early adulthood. Child Abuse \& Neglect, 32(6), 607-619. Retrieved from https://doi.org/10.1016/j.chiabu.2006.12.018

[6] Springer, K. W., Sheridan, J., Kuo, D., \& Carnes, M. (2007). Long-term physical and mental health consequences of childhood physical abuse: Results from a large population-based sample of men and women. child Abuse \& Neglect, 31(5), 517-530. Retrieved from https://doi.org/10.1016/j.chiabu.2007.01.003

[7] Schofield, T. J., Lee, R. D., \& Merrick, M. T. (2013). Safe, stable, nurturing relationships as a moderator of intergenerational continuity of child maltreatment: a meta-analysis. the Journal of Adolescent Health: Official Publication of the Society for Adolescent Medicine, 53(4 Suppl), S32-8. Retrieved from https://doi.org/ 10.1016/j.jadohealth.2013.05.004

[8] Gibb, B. E., Chelminski, I., \& Zimmerman, M. (2007). Childhood emotional, physical, and sexual abuse, and diagnoses of depressive and anxiety disorders in adult psychiatric outpatients. depression and Anxiety, 24(4), 256-263. Retrieved from https://doi.org/10.1002/da.20238

[9] Hildyard, K. L., \& Wolfe, D. A. (2002). Child neglect: developmental issues and outcomes $\hat{\sim}$. Child Abuse \& Neglect, 26(6), 679-695. Retrieved from

https://doi.org/10.1016/S0145-2134(02)00341-1

[10] Taillieu, T. L., Brownridge, D. A., Sareen, J., \& Afifi, T. O. (2016). Childhood emotional maltreatment and mental disorders: Results from a nationally representative adult sample from the United States. child Abuse \&amp ; Neglect, 59, 1-12. Retrieved from https://doi.org/10.1016/j.chiabu.2016.07.005

[11] Mersky, J. P., Topitzes, J., \& Reynolds, A. J. (2013). Impacts of adverse childhood experiences on health, mental health, and substance use in early adulthood: a cohort study of an urban, minority sample in the U.S. Child Abuse \& Neglect, 37(11), 917-925. Retrieved from https://doi.org/10.1016/j.chiabu.2013.07.011

[12] Gross, A. B., \& Keller, H. R. (1992). Long-term consequences of childhood physical and psychological maltreatment. Aggressive Behavior, 18(3), 171-185. retrieved from https://doi.org/10.1002/1098-2337(1992)18:3<171: :aid-ab2480180302>3.0.co;2-i

[13] Hoglund, C. L., \& Nicholas, K. B. (1995). Shame, guilt, and anger in college students exposed to abusive family environments. Journal of Family Violence, 10(2), 141-157. Retrieved from https://doi.org/10.1007/BF02110597

[14] Fairburn, C. G., Shafran, R., \& Cooper, Z. (1999). A cognitive behavioural theory of anorexia nervosa. Behaviour Research and Therapy, 37(1), 1-13. Retrieved from https://doi.org/ $\underline{10.1016 / \mathrm{s} 0005-7967(98) 00102-8}$

[15] Lampe, L., Slade, T., Issakidis, C., \& Andrews, G. (2003). Social phobia in the Australian National Survey of Mental Health and Well-Being (NSMHWB). Psychological Medicine, 33(4), 637-646. Retrieved from https://doi.org/10.1017/s0033291703007621

[16] Chu, L.-C. (2010). The benefits of meditation vis-à-vis emotional intelligence, perceived stress and negative mental health. International Society for the Investigation of Stress, 26(2), 169-180. Retrieved from https://doi.org/10.1002/smi.1289

[17] Greenberg, L. S. (2004). Emotion-focused therapy. Clinical Psychology \& Psychotherapy, 11(1), 3-16. Retrieved from https://doi.org/ 10.1002/cpp.388

[18] Toth, S. L., \& Manly, J. T. (2019). Developmental consequences of child abuse and neglect: Implications for intervention. Child Development Perspectives. Retrieved from 
https://srcd .onlinelibrary.wiley.com/doi/abs/10.11 $\underline{11 / \text { cdep. } 12317}$

[19] Toth, S. L., Petrenko, C. L. M., Gravener-Davis, J., \& Handley, E. D. (2016). Advances in prevention science: A developmental psy-chopathology perspective. in D. Cicchetti (Ed.), Developmental psy-chopathology (Vol. 4, In D. Cicchetti (Ed.), Developmental psy-chopathology (Vol. 4, pp. 815-873). New York, NY: Wiley

[20] Lieberman, A. F., Ghosh Ippen, C., \& Van Horn, P. (2015). "Don't hit my mommy!": A manual for child-parent psychotherapy with youngchildren exposed to violence and other trauma (2nd ed.). Washing-ton, DC: Zero to Three.

[21] Stevenson, J. (1999). The treatment of the long-term sequelae of child abuse. Journal of Child Psychology and Psychiatry, and Allied Disciplines, 40(1), 89-111 . Retrieved from https://www.ncbi.nlm.nih.gov/pubmed/10102727

[22] Moxley, K. M., Squires, J., \& Lindstrom, L. (2012). Early Intervention and Maltreated Children: A Current Look at the Child Abuse Prevention and Treatment Act and Part C. Infants and Young Children, 25(1), 3.
[23] Whipple, E. E., \& Richey, C. A. (1997). Crossing the line from physical discipline to child abuse: How much is too much? Child Abuse \& Neglect, 21(5), 431-444. retrieved from https://doi.org/10.1016/s0145-2134(97)00004-5 https://doi.org/10.1016/s0145-2134(97)00004-5

[24] Ip, P., Wong, R. S., Li, S. L., Chan, K. L., Ho, F. K., \& Chow, C.-B. (2016). Mental Health Consequences of Childhood Physical Abuse in Chinese Populations: A Meta-Analysis. Trauma, Violence \& Abuse, 17(5), 571-584. Retrieved from https://doi.org/10.1177/1524838015585317

[25] Rich, C. L., Gidycz, C. A., Warkentin, J. B., Loh, C., \& Weiland, P. (2005). Child and adolescent abuse and subsequent victimization: a prospective study. Child Abuse \& Neglect, 29(12), 1373-1394. retrieved from https://doi.org/10.1016/j.chiabu.2005.07.003

[26] Hu, Shuang. (2014). The impact of domestic violence on young children's mental health. Read Write Count (Teacher's Edition): Quality Education Forum (28), 78-78. 